\title{
SIADH associated with neuromyelitis optica involving hypothalamus.
}

\author{
Dimitris Papamargaritis ${ }^{1}$, Miles J Levy 1 , Narendra L Reddy 1 , Jithin George ${ }^{2}$, Ragini C Bhake \\ ${ }^{1}$ Department of Endocrinology, University Hospitals of Leicester NHS Trust, Leicester, UK \\ 2 Department of Neurology, University Hospitals of Leicester NHS Trust, Leicester, UK
}

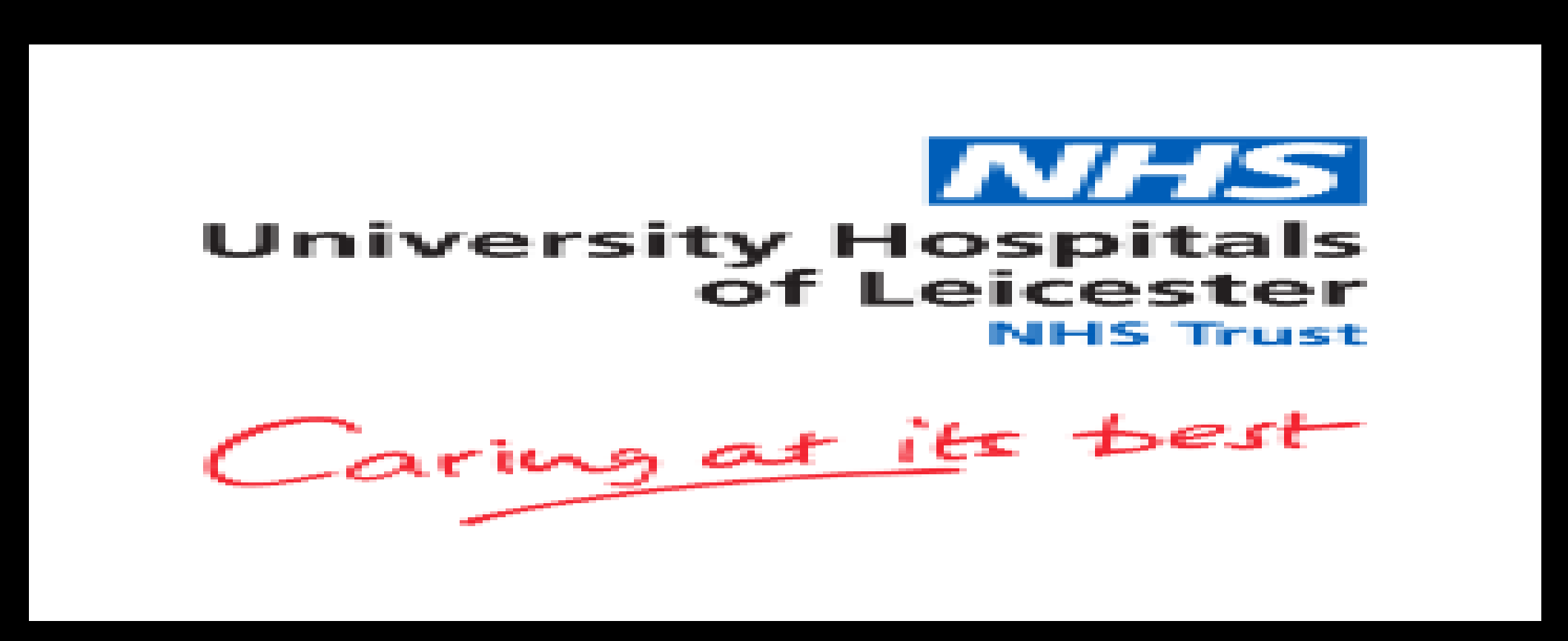

\section{Introduction}

- Syndrome of inappropriate antidiuretic hormone secretion (SIADH) is a cause of hyponatraemia which has been associated with many central nervous system (CNS) disorders . However, its association with neuromyelitis optica (NMO) or NMO spectrum disorders (NMOSD) is rare.

- NMO and NMOSD are inflammatory, autoimmune, demyelinating disorders of CNS predominantly affecting optic nerves and spinal cord but also certain brain regions. They are associated with the presence of $\operatorname{lgG}$ antibodies to aquaporin-4 (highly expressed in hypothalamus, brainstem, periventricle \& spine).

- We report here a case of NMOSD relapse which presented with hyponatraemia due to SIADH.

\section{Case report}

- A 21-year old Asian woman presented and admitted to the hospital with possible relapse of NMOSD (initial diagnosis at the age of 16 and confirmed positive antiaquaporin-4 antibodies). She had headache, dizziness, right hand weakness and severe hyponatraemia (serum Na 116 mmol/l [135-145])

- Emesis, dominant in previous relapses was absent and patient was euvolaemic.

- Medications included Prednisolone 20mg OD, Azathioprine, Proton Pump inhibitor (PPI).

- Investigations demonstrated serum osmolality $\left(\mathrm{Se}_{\mathrm{osm}}\right)$ was $250 \mathrm{mOsm} / \mathrm{Kg}$, urine osmolality $\left(\mathrm{U}_{\text {osm }}\right) 468 \mathrm{mOsm} / \mathrm{Kg}$, FT4: 14 (normal range $\left.9-25\right)$, TSH 2.7 (normal range $0.3-5.0)$. A diagnosis of SIADH secretion was made based on the above results.

- Hyponatremia improved to $129 \mathrm{mmol} / \mathrm{l}$ in 2 days with fluid restriction and conversion of PPI to histamine-2-receptor antagonist. Patient was discharged from the hospital with further follow-up appointments in the Endocrine and Neurology clinic as outpatient.

\section{Further relapse and management}

- 2-weeks later patient reviewed in the Endocrine clinic where she reported increasing somnolence, increased frequency of urination without evidence of infection, and unchanged thirst.

- Documented fluid balance over a 24-hour period was normal as were repeat paired serum and urine test results $\left(\mathrm{Na}^{+} 141, \mathrm{Se}_{\mathrm{osm}} 293, \mathrm{U}_{\mathrm{osm}} 698\right)$.

- She appeared emotionally labile, expressed suicidal ideation, and fell into deep sleep mid-consultation precipitating urgent admission for treatment of further relapse with 5 days of IV methylprednisolone (1g once daily).

- Somnolence - a common symptom on NMOSD relapse - was likely secondary to central sleep apnoea confirming neurological progression as sodium was normal.

- MRI brain performed at this admission showed: "T2/FLAIR hyperintensity with associated contrast enhancement affecting the medial right thalamus/hypothalamus and left hypothalamus. Findings in keeping with active NMO."

- Symptoms improved with IV methylprednisolone and patient was symptom free and discharged after 5 days, on high dose of prednisolone. Patient was also offered treatment with Rituximab at the Neurology clinic which she is considering.

Table 1. Biochemical values

\begin{tabular}{|c|c|c|c|c|c|}
\hline & 10:5/17 & $13 / 5 / 17 / 8]$ & 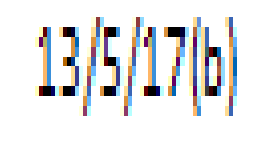 & $14 / 5 / 17$ & $15 / 5 / 177$ \\
\hline 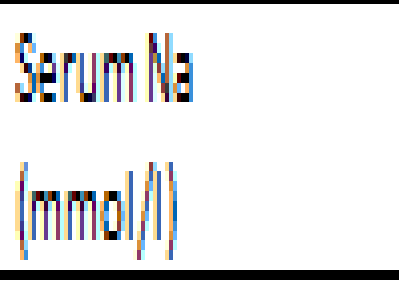 & 134 & 1.18 & 1.16 & 129 & 130 \\
\hline 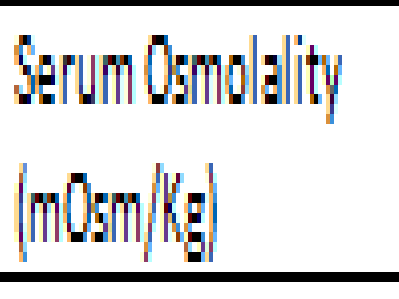 & & & 250 & 270 & \\
\hline 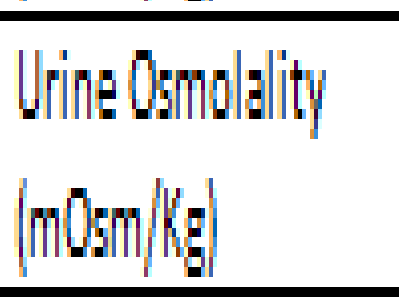 & & & & & 468 \\
\hline TiH & 2.7 & & & & \\
\hline
\end{tabular}

Figure 1. MRI brain - SAG T2 FLAIR

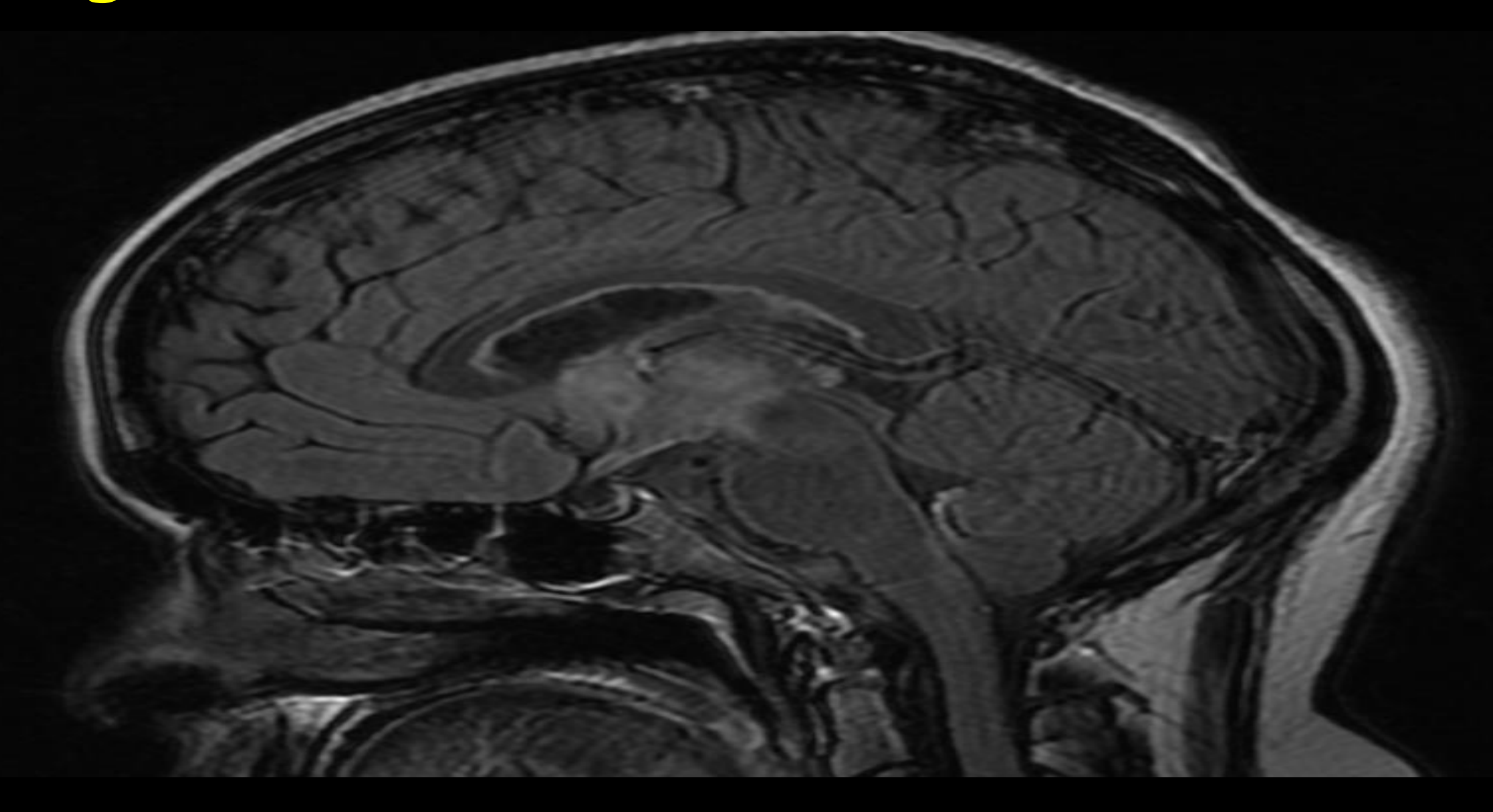

Discussion
Figure 2. MRI brain - Axial T2 TSE

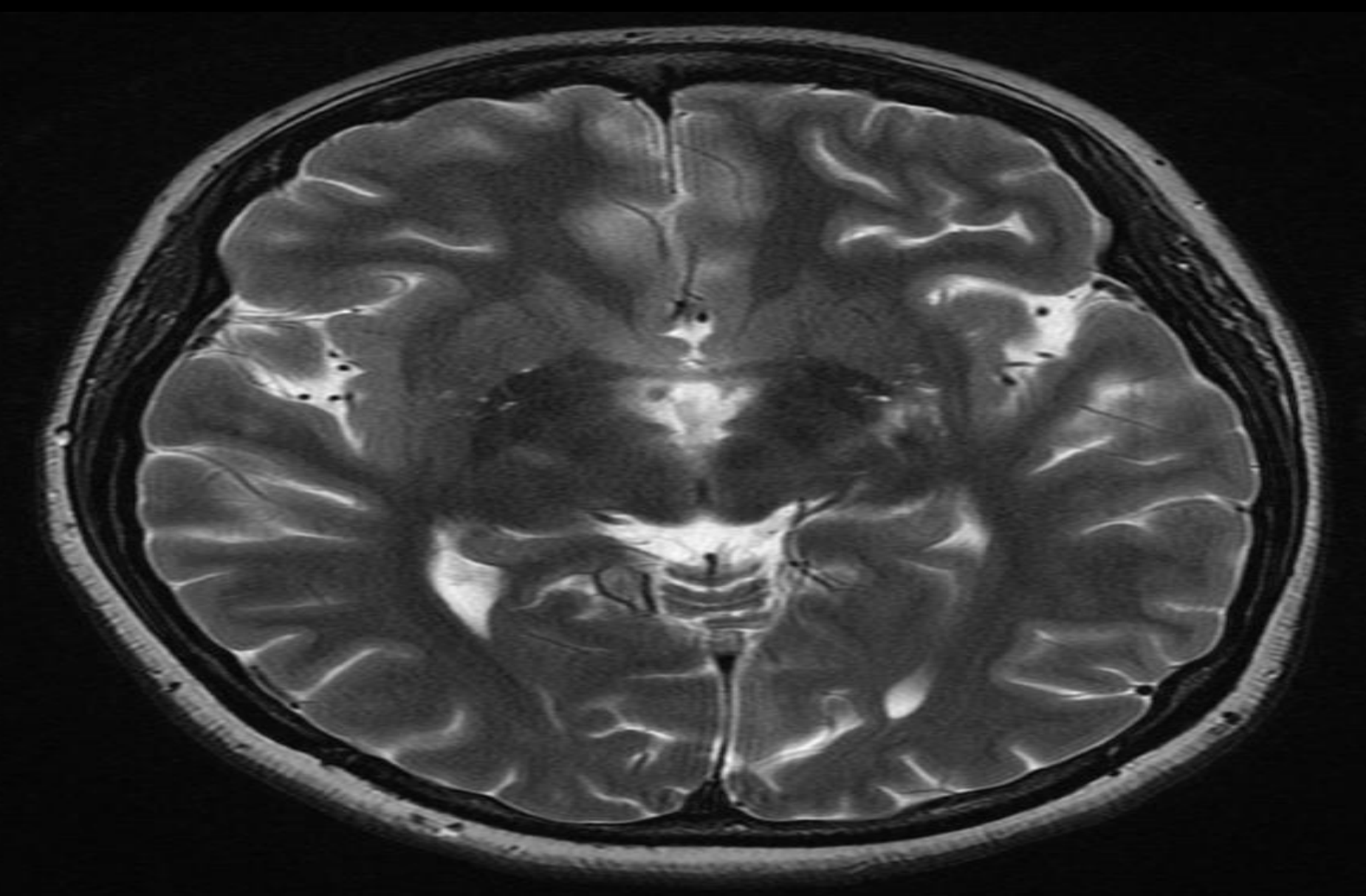

- Previous case series have shown that approximately $15 \%$ of patients with NMO/NMOSD and positive IgG antibodies for aquaporin-4 meet the criteria for SIADH1,2

- Hypothalamic lesions may be common in patients with SIADH on background of NMO with positive anti-aquaporin-4 antibodies ${ }^{1}$.

- SIADH may reportedly precede an exacerbation of NMO, or accompany \& resolve after a relapse.

- Unlike autoimmune idiopathic hypophysitis which typically causes central-Diabetes Insipidus, SIADH has been reported more commonly with NMO.

- Increasing awareness of NMO-associated-endocrinopathy, especially of SIADH is needed. Other endocrinopathies associated with NMO/NMOSD include type 2 diabetes mellitus, diabetes insipidus, hyperprolactinaemia with or without galactorrhoea, hyperphagia and consequent obesity, amenorrhoea and hypothyroidism.

\section{References}

1. Pu S, Long Y, Yang N, He Y, Shan F, Fan Y, Yin J, Gao Q, Cong G. Syndrome of inappropriate antidiuretic hormone secretion in patients with aquaporin-4 antibody. J Neurol. 2015 Jan;262(1):101-7.

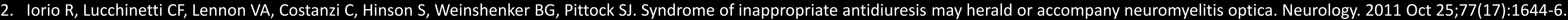

